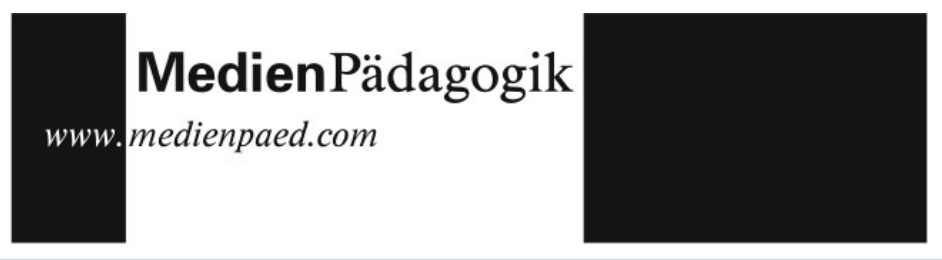

Rezensionen
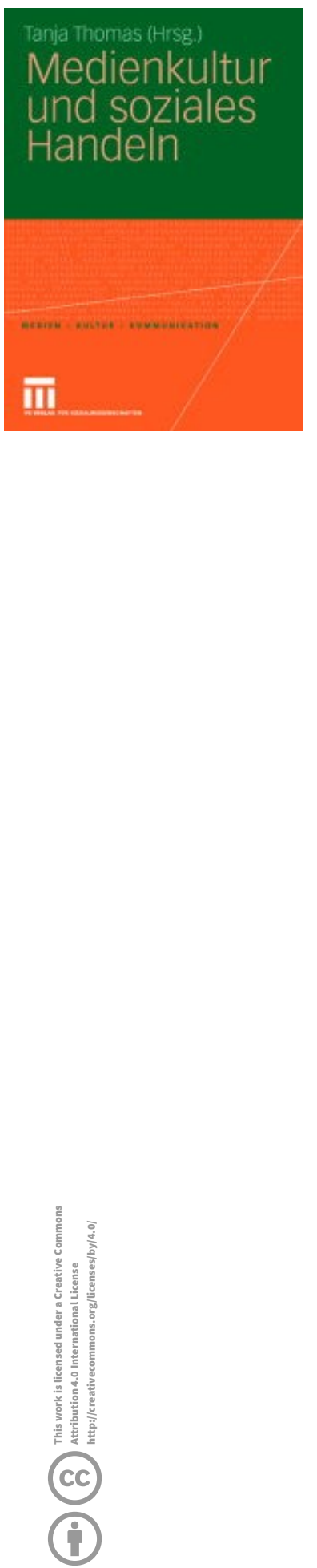

Tanja Thomas

Medienkultur und soziales Handeln - Eine Einführung

Wiesbaden: VS Verlag für Sozialwissenschaften, 2009. 321 Seiten

ISBN 3-531-15128-1

$€ 29,90 ;$ CHF 51,00

\section{Theoretische und empirische Facetten des komplexen Beziehungsgeflechts Medienkultur und soziales Handeln}

Wie häufig schon haben wir gehört oder gelesen, dass der Altag der Menschen moderner Gesellschaften von Medien durchdrungen ist? Wie selbstverständlich ist allmählich die Trias «Individuum - Medien - Gesellschaft»? Gleichwohl besteht immer noch großer Klärungs- und Erkenntnisbedarf, wenn es um ein genaues und differenziertes Verstehen und um ein Verständnis der Konsequenzen der «Mediatisierung des Alltags» geht. In dem vorliegenden Sammelband beschäftigen sich 17 Autoren bzw. Autorinnen aus soziologischer und kommunikationswissenschaftlicher Perspektive mit der Beschreibung und Analyse des komplexen Verhältnisses von Medienkultur und sozialem Handeln. Dabei geht es innen vor allem darum, die Begriffe «Medienkultur» und "Mediatisierung» für verschiedene Fragestellungen und Ereignisse zu operationalisieren und voneinander abzugrenzen. Insbesondere die ersten drei Beiträge von Tanja Thomas, Friedrich Krotz und Andreas Hepp widmen sich Begriffsbestimmungen und der Theorieentwicklung, wobei sie an eigene Vorarbeiten anschließen oder - wie Hepp - sich an klassischen und zeitgenössischen Konzepten u.a. von Manuel Castells orientieren und diese um eigene Modelle erweitern. Die darauffolgenden Beiträge sind dann thematisch weit gestreut und größtenteils empirisch (qualitativ) ausgerichtet. Es finden sich u.a. Beiträge zum alltäglichen Medienhandeln junger Paare via Mobiltelefon, Internet und Fernsehen, zur Bedeutung der Dramatisierung des Altags in populären TV-Formaten (wie etwa Casting-Shows), zur Medialisierung religiösen Handelns am Beispiel des 20. Weltjugendtages. Es werden historische populäre Jugendkulturen mit ihrem spezifischen Mediengebrauch vorgestellt und auch der Wandel der japani- 


\section{Rezensionen}

schen Jugendszene Visual kei nachgezeichnet, die sich internetbasiert präsentiert und global verbreitet. Des Weiteren werden aktuelle Körperpraktiken und -inszenierungen vor dem Hintergrund gesellschaftlicher und medienkultureller Entwicklungen diskutiert. Außerdem widmen sich verschiedene Autoren der Werbe- und TV-Spielkultur sowie neuen Formen von Politikvermittlung am Beispiel der Sendung TV total. Der Band schließt wiederum mit einer umfänglichen soziologischen Auseinandersetzung um die sozialen Phänomene «Kritik», «Widerstand» und «Gegenkultur», die zu unterschiedlichen Zeiten und mittels verschiedener Medien jeweils andere Kollektivierungs- und Mobilisierungspotenziale besaßen. Wie diese komprimierte Inhaltsangabe schon verdeutlicht, ist der Sammelband ausgesprochen facettenreich. Allerdings haben sich alle Autoren und Autorinnen sehr bemüht, den übergeordneten Themenbereich «Medienkultur und soziales Handeln» mit Bezug auf ihre jeweilige disziplinäre Verortung und ihre eigenen Arbeitsund Forschungsbereiche angemessen zu verhandeln. Erfreulich ist, dass ein Buch vorliegt, welches die wesentlichen Vertreter und Vertreterinnen des Diskurses vereint. Wer die Debatte um den Begriff der Mediatisierung schon länger verfolgt, wird auf bekannte Erkenntnisse stoßen, gleichwohl aber seinen Wissensstand insbesondere um vielfältige Forschungsergebnisse erweitern können. Wer sich dem Thema erst annähern möchte, wird vermutlich ohne profunder mediensoziologischer oder kulturwissenschaftlicher Vorkenntnisse mit den unterschiedlichen Konzeptionalisierungsversuchen zu Beginn des Buches etwas überfordert sein. Folglich ist das Buch eher für Studierende zu empfehlen, die in ihrem Studium der Medien-, Kommunikations- bzw. Kulturwissenschaft, Soziologie oder Pädagogik schon fortgeschritten sind und selbstverständlich den in Wissenschaft und Forschung Tätigen, die an den aktuellen Stand der Diskussion anknüpfen möchten.

Dagmar Hoffmann 